\title{
Erratum: Doppler Broadening of In-Flight Positron \\ Annihilation Radiation due to Electron Momentum \\ [Phys. Rev. Lett. 86, 5612 (2001)]
}

\begin{abstract}
A. W. Hunt, D. B. Cassidy, P. A. Sterne, T. E. Cowan, R. H. Howell, K. G. Lynn, and J. A. Golovchenko (Published 10 September 2001)
\end{abstract}

DOI: $10.1103 /$ PhysRevLett.87.139901

PACS numbers: 78.70.Bj, 41.75.Ht, 71.20.-b, 99.10.+g

The last author's name in our Letter, J. A. Golovchenko, was misspelled. The correct author list is shown above. The Physical Review Letters on-line version was corrected on 23 August 2001. 